\title{
Oral intake of hydrogen-rich water inhibits intimal hyperplasia in arterialized vein grafts in rats
}

\author{
Qiang Sun 1,2,3, Tomohiro Kawamura ${ }^{1,4}$, Kosuke Masutani ${ }^{5}$, Ximei Peng ${ }^{1}$, Qing Sun ${ }^{2}$, \\ Donna B. Stolz ${ }^{6}$, John P. Pribis 7 , Timothy R. Billiar ${ }^{7}$, Xuejun Sun ${ }^{2}$, \\ Christian A. Bermudez ${ }^{1}$, Yoshiya Toyoda ${ }^{1,4}$, and Atsunori Nakao ${ }^{1,4,7 *}$
}

\begin{abstract}
${ }^{1}$ Department of Cardiothoracic Surgery, University of Pittsburgh Medical Center, Pittsburgh, PA, USA; ${ }^{2}$ Department of Diving Medicine, Faculty of Naval Medicine, Second Military Medical University, Shanghai 200433, PR China; ${ }^{3}$ Naval Medical Research Institute, Shanghai 200433, PR China; ${ }^{4}$ Thomas E. Starzl Transplantation Institute, University of Pittsburgh Medical Center, E1551, Biomedical Science Tower, 200 Lothrop Street, Pittsburgh, PA, USA; ${ }^{5}$ Department of Pathology, University of Pittsburgh Medical Center, Pittsburgh, PA, USA; ${ }^{6}$ Center for Biologic Imaging, University of Pittsburgh Medical Center, Pittsburgh, PA, USA; and ${ }^{7}$ Department of Surgery, University of Pittsburgh Medical Center, Pittsburgh, PA, USA
\end{abstract}

Received 4 August 2011; revised 18 January 2012; accepted 20 January 2012; online publish-ahead-of-print 27 January 2012

Time for primary review: 28 days

\begin{abstract}
Aims
Arterialized vein grafts often fail due to intimal hyperplasia. Hydrogen potently protects organs and cells from many insults via its anti-inflammatory and antioxidant properties. We investigated the efficacy of oral administration of hydrogen-rich water (HW) for prevention of intimal hyperplasia.

Methods and results

The inferior vena cava was excised, stored in cold Ringer solution for $2 \mathrm{~h}$, and placed as an interposition graft in the abdominal aorta of syngeneic Lewis rats. HW was generated by immersing a magnesium stick in tap water $\left(\mathrm{Mg}+2 \mathrm{H}_{2} \mathrm{O} \rightarrow \mathrm{Mg}(\mathrm{OH})_{2}+\mathrm{H}_{2}\right)$. Beginning on the day of graft implantation, recipients were given tap water [regular water (RW)], HW or HW that had been subsequently degassed water (DW). Six weeks after grafting, the grafts in the rats given RW or DW had developed intimal hyperplasia, accompanied by increased oxidative injury. HW significantly suppressed intimal hyperplasia. One week after grafting, the grafts in HW-treated rats exhibited improved endothelial integrity with less platelet and white blood cell aggregation. Up-regulation of the mRNAs for intracellular adhesion molecules was attenuated in the vein grafts of the rats receiving HW. Activation of p38 mitogen-activated protein kinase, matrix metalloproteinase (MMP)-2, and MMP-9 was also significantly inhibited in grafts receiving $\mathrm{HW}$. In rat smooth muscle cell $(\mathrm{A} 7 \mathrm{r} 5)$ cultures, hydrogen treatment for $24 \mathrm{~h}$ reduced smooth muscle cell migration.
\end{abstract}

Keywords

\section{Conclusion \\ Drinking HW significantly reduced neointima formation after vein grafting in rats. Drinking HW may have therapeutic value as a novel therapy for intimal hyperplasia and could easily be incorporated into daily life.}

\section{Introduction}

Arterial bypass surgery with autologous vein grafts is a common operation for treatment of occluded coronary arteries or occluded arteries in the extremities and for the creation of arteriovenous fistulas for blood access in patients who require haemodialysis. ${ }^{1-3} \mathrm{Al}-$ though bypass surgery with venous conduits is an effective treatment and gives satisfactory early results, the frequency of graft failure is unexpectedly high. Intimal hyperplasia $(\mathrm{IH})$, characterized by the accumulation of smooth muscle cells (SMCs) and extracellular matrix (ECM) in the intima of the vein graft, is the most common cause of limited patency. $\mathrm{IH}$ occurs in $\sim 50 \%$ of vein grafts and is a major cause of recurrent ischaemia. ${ }^{4,5}$

$\mathrm{IH}$ is a universal response of vein grafts to altered haemodynamic forces and hypoxia-reoxygenation injury. The ischaemic period during harvesting also contributes to local injury of the vein graft. $^{6}$ Direct or indirect graft tissue injury, due to free radical species generated during the vein grafting process, leads to the release of cytokines and chemokines, and is one of the critical events in the pathophysiology of $\mathrm{IH}$ in vein grafts. ${ }^{7,8}$ After engraftment, the vein graft is subject to suddenly increased mechanical stretch due to exposure to arterial blood pressure. This stretch induces rapid cell death with

* Corresponding author. Tel: +1 412648 9547; fax: +1 412624 6666, Email: anakao@imap.pitt.edu

Published on behalf of the European Society of Cardiology. All rights reserved. (C) The Author 2012. For permissions please email: journals.permissions@oup.com. 
a reduction in cell density by $\sim 60 \%$ within the first day after surgery and is followed by an increase in abnormal proliferation of the cells. ${ }^{9}$ Vein grafts are exposed to an environment with approximately threefold higher oxygen concentration than the venous circulation, and this environment promotes oxidative injury within 2 weeks. ${ }^{10,11}$ In addition, macrophages infiltrate the graft after implantation, followed by activation and production of reactive oxygen species (ROS). Excessive ROS production overwhelms the endogenous antioxidant defence mechanisms and leads to the oxidation of macromolecules, such as DNA, proteins, and lipids.

Hydrogen functions as an important physiologic regulatory factor with antioxidant, anti-inflammatory, and antiapoptotic protective effects on cells and organs, and the ability to mitigate a variety of diseases. ${ }^{12-15}$ Although the safety of inhaled hydrogen gas in humans is demonstrated by its application in Hydreliox, an exotic breathing gas mixture containing $49 \%$ hydrogen used during very deep technical diving, ${ }^{16}$ hydrogen inhalation therapy is still in the preclinical stages of investigation. Currently, administering hydrogen by inhalation is not practical or suitable for continuous consumption for preventative uses. On the other hand, solubilized hydrogen is a portable, easily administered, and safe method of delivering hydrogen to humans. ${ }^{15,17-20}$

Based on the potent antioxidant and anti-inflammatory properties of hydrogen, we hypothesized that oral intake of hydrogen-rich water (HW) could act as a novel and effective approach to prevent the development of $\mathrm{IH}$. We tested our hypothesis using an established rat vein-grafting model with oral administration of $\mathrm{HW}^{6,21}$ Because the long-term patency of vein grafts must be improved, studies of feasible therapeutic strategies to prevent $\mathrm{IH}$ are warranted.

\section{Methods}

\subsection{Generation of hydrogen-rich water and control water}

The magnesium stick used to produce hydrogen is a plastic-shelled product consisting of metallic magnesium (99.9\% pure) and natural stones combined with ceramics in a polypropylene container (Doctor SUISOSUI ${ }^{\circledR}$, Friendear, Tokyo, Japan). ${ }^{19}$ Hydrogen is generated when the stick is placed in drinking water for $24 \mathrm{~h}\left(\mathrm{Mg}+2 \mathrm{H}_{2} \mathrm{O} \rightarrow \mathrm{Mg}\right.$ $\left.(\mathrm{OH})_{2}+\mathrm{H}_{2}\right) .{ }^{15}$ The hydrogen concentration in the water bottle was monitored using a hydrogen needle sensor (DHS-001, ABLE, Tokyo, Japan). Hydrogen concentration was maintained at levels between 0.55 and $0.65 \mathrm{mM}$ and $\mathrm{pH}$ ranged from 9.2 to 9.9. Control rats received either unmodified tap water (RW) or water was supplemented with hydrogen using the magnesium stick and then subsequently degassed by exposure to air for $48 \mathrm{~h}$ (DW) (Table 1). Water was replaced with freshly prepared HW or DW every $24 \mathrm{~h}$.

Table I Drinking water for each experimental group

\begin{tabular}{|c|c|c|c|c|}
\hline Drinking water & Mg stick & $\begin{array}{l}\text { Mg conc } \\
(\mathrm{mg} / \mathrm{L})\end{array}$ & $\begin{array}{l}\mathrm{H}_{2} \text { conc } \\
(\mathrm{mM})\end{array}$ & pH \\
\hline Regular water (RW) & No & $<1.0$ & 0 & $6.8-7.1$ \\
\hline Degassed water (DW) & Yes & $6.2-7.9$ & 0 & $9.2-9.9$ \\
\hline Hydrogen water (HW) & Yes & $6.1-7.9$ & $0.55-0.65$ & $9.3-9.8$ \\
\hline
\end{tabular}

\subsection{Animals}

Inbred male Lewis (LEW RT.1') rats weighting 200-250 g (Harlan Sprague Dawley, Inc., Indianapolis, IN, USA) were housed in conventional animal facilities with 12:12 light/dark cycle. All procedures were performed in accordance with the guidelines of the Institutional Animal Care and Use Committee at the University of Pittsburgh and the Guide for the Care and Use of Laboratory Animals published by the US National Institutes of Health (NIH Publication No. 85-23, revised 1996).

\subsection{Determination of hydrogen concentration in blood}

RW, DW, or HW (3 mL) was orally administered by gavage to naive Lewis rats. Prior to administration and 15, 30, and 60 min after oral intake, arterial blood and venous blood were taken from femoral artery and vein, respectively. Blood was placed in a vacuum glass tube and air-phase hydrogen levels were measured by gas chromatography (Biogas analyzer BAS-1000, Mitleben, Osaka, Japan). ${ }^{17,22}$

\subsection{Vein grafting}

Both donors and recipients were anaesthetized with inhaled isoflurane (2\%). Toe pinch reflex, muscular relaxation, and respiration rates were monitored to determine that adequate anaesthesia was administered. Under general anaesthesia, a 20 -mm-long segment of inferior vena cava was excised and preserved in lactated Ringer (LR) solution at $4^{\circ} \mathrm{C}$ for $2 \mathrm{~h}$. After the recipients were anaesthetized, the vein graft was interposed into the recipient's abdominal aorta using microsurgical techniques, and the aorta of the recipients was then ligated between the two anastomoses. $^{21,23}$

\subsection{Treatment protocols}

The vein graft recipients were randomly placed in treatment groups and given different types of drinking water ad libitum, starting immediately after surgery and continuing until tissue collection. The treatment groups were: RW, vein graft recipients that consumed regular tap water after vein grafting; DW, vein grafts recipients that consumed DW after vein grafting; and HW, vein grafts recipients that consumed hydrogensupplemented water after vein grafting. A sham group, in which the naïve vein was harvested from unoperated LEW rats, was also included. Animals were humanely euthanized using an overdose of isoflurane (4\%) and samples were collected by thoracotomy 1 week $(n=5$ for each group) or 6 weeks ( $n=6$ for each group) after grafting. The middle part of each vein graft sample $(\sim 1 \mathrm{~cm})$ was harvested, to avoid foreign body reaction at the anastomoses at both ends, and cut in three cross-sectional pieces, which were then processed for histology or snapfrozen in liquid nitrogen and kept at $-80^{\circ} \mathrm{C}$ until analysis.

\subsection{Histomorphometric analysis}

We manually perfused vein grafts harvested 6 weeks after grafting with $40 \mathrm{~mL}$ LR solution for $1 \mathrm{~min}$ via the puncture of the heart. We confirmed that complete circles were present in the cross-sectional vein samples in all experimental groups, fixed the samples in 10\% formalin after and embedded them in paraffin at room temperature. Four sections per samples were examined from each graft. To evaluate the development of $\mathrm{IH}$, the slides were stained with haematoxylin and eosin, Verhoeff Van-Gieson (Rowley Biochemical Institute, Danvers, MA, USA), or Masson trichrome (Rowley Biochemical Institute). Staining for smooth muscle actin ( $\alpha$-SMA) was performed using anti- $\alpha$-SMA antibodies (DAKO, Carpinteria, CA, USA), followed by incubation with biotinylated secondary antibodies (DAKO). The immune complexes were visualized with 3-animo-9-ethyl carbazole and haematoxylin counterstaining. Images of the vessel crosssections were taken using a Zeiss microscope (Axioskop, lowa City, IA, USA) and placed on a colour scanner (EPSON Smart Panel, Fremount, CA, USA). After digitized images were obtained, areas were measured 
by computer planimetry using NIH Image (v1.62) and expressed as the percent area of intima/intima + media in each cross-section.

To investigate tissue oxidation, the vein graft samples were stained with anti-8-hydroxy-2'-deoxyguanosine (8-OHdG; OxisResearch, Portland, OR, USA), anti-nitrotyrosine (Santa Cruz Biotechnology, Santa Cruz, CA, USA), and anti-4-hydroxy-2-nonenal (4-HNE; JAICA, Shizuoka, Japan). Macrophage infiltration was assessed by anti-rat CD68 staining (ED-1, Serotec, Raleigh, NC, USA). For each slide, two investigators independently reviewed five random fields containing endothelium, with the sample identities masked. Cells positive for 8-OHdG and ED-1 were counted in each high-power field $(\times 400)$. The intensity of nitrotyrosine or $4 \mathrm{HNE}$ stain was quantitatively scored on a scale from 1 to 5 , as described previously, ${ }^{8}$ and the scores were averaged.

\subsection{Scanning electron microscopy}

After treatment with RW, DW, or HW for 1 week, a cross-sectional piece from the middle of each vein graft was fixed in $2.5 \%$ glutaraldehyde for $1 \mathrm{~h}$ and pinned open on silicone rubber. The tissue was post-fixed in aqueous $1 \%$ OsO4 for $1 \mathrm{~h}$. After PBS washes, the tissue was dehydrated through a graded series of $30-100 \%$ ethanol, dried in a critical point dryer, mounted to metal stubs, and sputter coated with $3.5 \mathrm{~nm}$ coating of gold-palladium. The endothelial surfaces in each vein graft were reviewed by one of the authors (D.B.S.) with the sample identities masked, and typical features of luminal surfaces were digitally imaged with a JEOL JEM-6335F scanning electron microscope (JEOL Peabody, MA, USA).

\subsection{Total RNA extraction and SYBR green real-time PCR}

The mRNA levels for endothelin receptor-A, intercellular adhesive molecule-1 (ICAM-1), tumour necrosis factor- $\alpha$ (TNF- $\alpha$ ), and interleukin-6 (IL-6) were quantified in duplicate samples using SYBR Green two-step, real-time PCR using SYBR Green PCR Master Mix (PE Applied Biosystems, Foster City, CA, USA), as previously described. ${ }^{21}$ Each sample was analysed in duplicate using the conditions recommended by the manufacturer.

\subsection{Western blotting}

Protein extracts from snap-frozen vein graft samples were separated on sodium dodecyl sulfate-polyacrylamide gels and electroblotted to polyvinylidene difluoride membranes. Phosphorylated p38 mitogen-activated protein kinase (MAPK), total P38MAPK, phosphorylated extracellular signal-regulated kinase (ERK) $1 / 2$, total ERK1/2, matrix metalloproteinase (MMP)-2, MMP-9, and $\beta$-actin were detected with monoclonal antibodies (all from Cell Signaling Technology, Beverly, MA, USA) and appropriate secondary antibodies. Membranes were developed with the Supersignal detection system (Pierce Chemical, Rockford, IL, USA) and were exposed to film.

\subsection{Enzymatic activities of MMP-2 and MMP-9}

Enzymatic activities of MMP-2 and MMP-9 were determined using a Novex ${ }^{\circledR} 10 \%$ zymogram gel (Invitrogen, Carlsbad, CA, USA). Vein graft samples are denatured in SDS buffer under non-reducing conditions and without heating, and run on the zymogram gel using Tris-Glycine SDS running buffer. After electrophoresis, the proteins were renatured by incubating the gel in renaturing buffer containing a non-ionic detergent for $30 \mathrm{~min}$. The gels are then equilibrated in developing buffer, stained with fresh coomassie blue, and destained with methanol. Band intensity, indicating regions of protease activity, was measured using Image J software (http://rsb.info.nih.gov/ij/).

\subsection{Hydrogen-rich medium production}

Hydrogen was dissolved in DMEM for $6 \mathrm{~h}$ under high pressure $(0.4 \mathrm{MPa})$ to a supersaturated level. Hydrogen-rich medium was stored at atmospheric pressure at $4^{\circ} \mathrm{C}$ in an aluminum bag with no dead volume, sterilized by gamma radiation, and freshly prepared once a week to ensure that the hydrogen concentration was maintained at $0.6 \mathrm{mmol} / \mathrm{L}$. The hydrogen content of the medium was confirmed using the gas chromatography method described by Ohsawa et al. ${ }^{13}$

\subsection{Wound migration assay}

The A7r5 rat aortic SMC line (cell bank, Chinese Academy of Sciences) was cultured to $100 \%$ confluence. The cultures were incubated with hydrogen-rich medium or control media, with or without $10 \%$ foetal bovine serum (Invitrogen), for $6 \mathrm{~h}$ before wounding. Scratch 'wounds' were made with a $200 \mu \mathrm{L}$ pipette tip and photographs were taken immediately (time zero) and $24 \mathrm{~h}$ after wounding. The media were changed every $6 \mathrm{~h}$ to maintain the concentration of hydrogen at $0.6 \mathrm{mmol} / \mathrm{L}$. Cell migration to close the wounded area was measured. Results were expressed as a migration index, the distance migrated by the cells cultures in hydrogen-rich medium relative to the distance migrated by cells cultured in regular medium. Experiments were carried out in triplicate and repeated at least three times.

\subsection{Cell proliferation assay}

A7r5 cell suspension was seeded in 96 -well plates $\left(5 \times 10^{3}\right.$ cells per well $)$ and incubated for up to $72 \mathrm{~h}$. Medium containing $10 \%$ foetal bovine serum was changed every $6 \mathrm{~h}$ to maintain the concentration of hydrogen at $0.6 \mathrm{mmol} / \mathrm{L}$. Cell proliferation was analysed using a WST-8 Cell Counting Kit-8 (CCK-8, Beyotime, Jiangsu, China) according to the manufacturer's protocol. $^{24,25}$

\subsection{Oxidative damage in culture and lactate dehydrogenase leakage assay}

Human umbilical vein endothelial cells (HUVEC, Sciencecell) were seeded into 96-well plates $12 \mathrm{~h}$ before treatment. Oxidative damage was induced by incubation with hydrogen peroxide $\left(\mathrm{H}_{2} \mathrm{O}_{2}\right)$. During treatment, the cells were exposed to DMEM, DMEM with $100 \mu M \mathrm{H}_{2} \mathrm{O}_{2}$ or hydrogen-rich DMEM with $100 \mu \mathrm{M} \mathrm{H}_{2} \mathrm{O}_{2}$ and $0.6 \mathrm{mmol} / \mathrm{L} \mathrm{H}_{2}$ for $2 \mathrm{~h}$. Cell viability was determined by the CCK-8 assay. A lactate dehydrogenase (LDH) leakage assay was used to examine plasma membrane integrity. For the LDH leakage assay, HUVEC were seeded into 6-well plates $12 \mathrm{~h}$ before

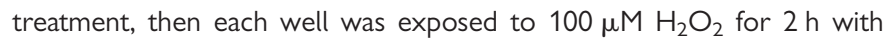
or without hydrogen-rich medium as described earlier. The culture fluid was collected and LDH leakage was detected using an LDH cytotoxicity detection kit (Nanjing KeyGen Biotech. Co. Ltd, China), according to the manufacturer's protocol.

\subsection{Malondialdehyde assay}

Malondialdehyde (MDA), a non-specific marker of lipid peroxidation, was measured in cultured HUVECs and in the supernatant of homogenized vein graft tissue. HUVECs were seeded into 6 -well plates $12 \mathrm{~h}$ before treatment. After treatment, cells were disrupted by Western/IP Cell Disruption Fluid $(0.1 \mathrm{~mL} /$ well). The fluid was collected and tested using an MDA detection kit (Beyotime Institute of Biotechnology, Haimen, China) according to the manufacturer's protocol. MDA was measured in the supernatant of homogenized vein graft tissue using the Bioxytech MDA-586 kit (Oxis Research, Portland, OR, USA) according to the manufacturer's protocol.

\subsection{Statistical analysis}

Values are presented as mean \pm standard deviation. Statistical analysis was done using SPSS Statistics 17.0 (SPSS, Inc., Chicago, IL, USA). Differences between groups were determined with a one-way ANOVA followed by 


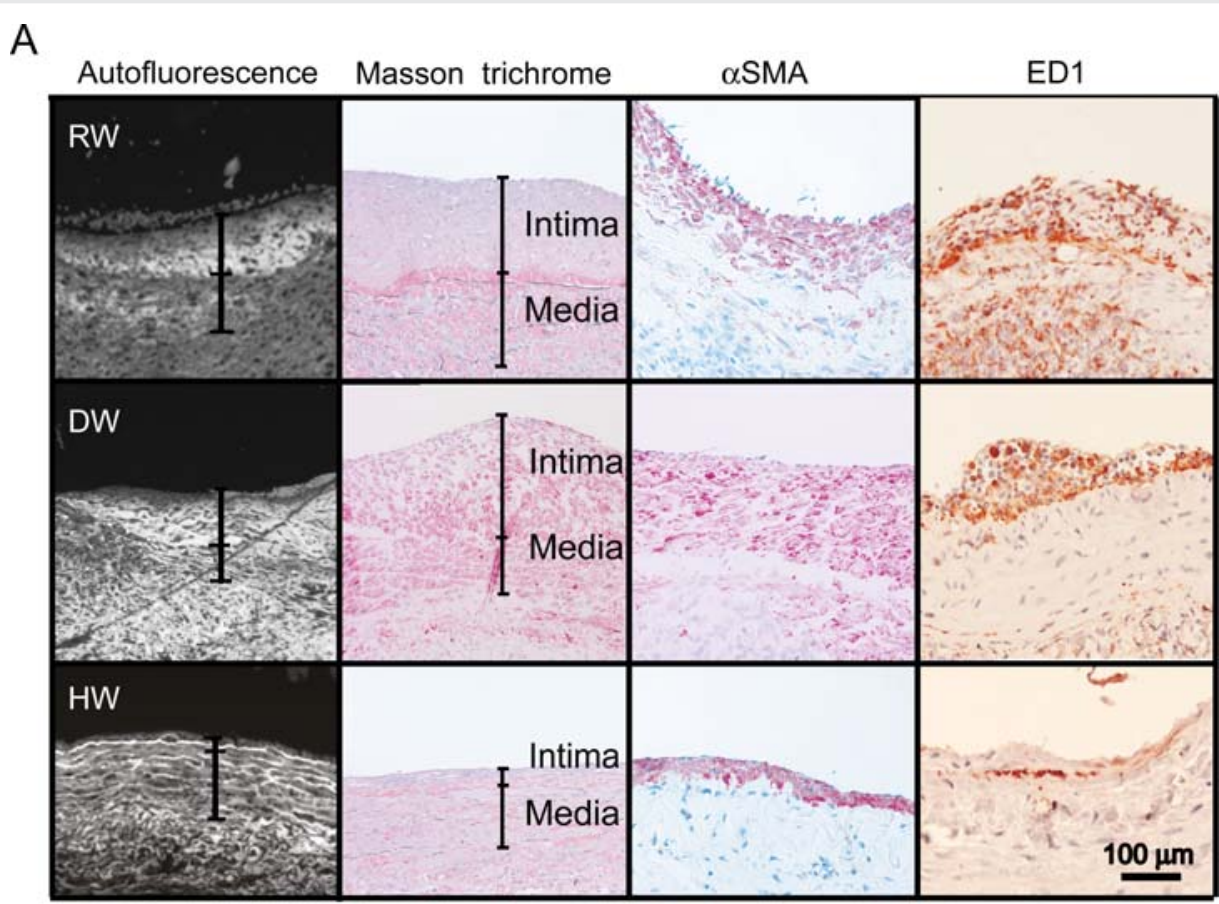

B

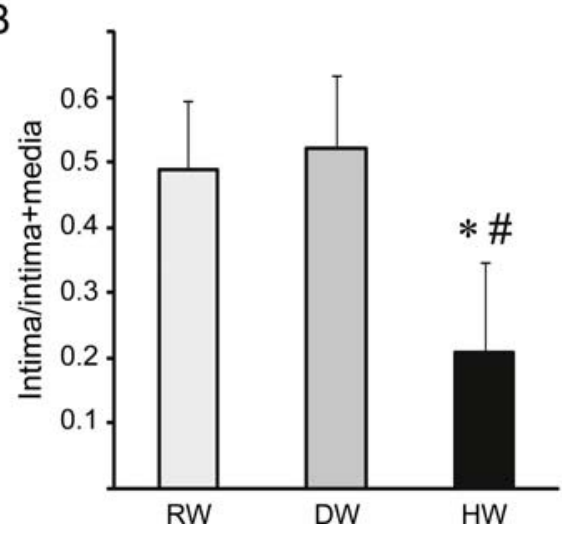

C

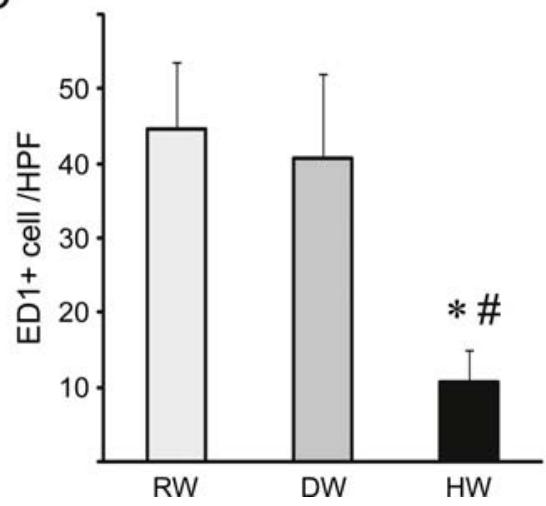

Figure I (A) Morphological assessment of arterialized vein grafts 6 weeks after engraftment. Vein grafts from rats that received regular water (RW), degassed water (DW), or hydrogen-rich water (HW). Representative images of tissue autofluorescence, Masson trichrome stain and immunohistochemical staining for $\alpha$-SMA or ED-1 are shown. (B) Histogram quantifying intimal area/intimal + medial area in the vein grafts. (C) Macrophage infiltration as assessed by ED-1 staining. HPF, high power field ( $n=6$ for RW, $n=5$ for DW and $n=6$ for HW; 5 HPF were counted per sample; $* P<0.05$ vs. RW, ${ }^{\#} P<0.05$ vs. DW).

Student-Newman-Keuls tests. $P$-values $<0.05$ were considered statistically significant.

\section{Results}

\subsection{Oral administration of HW increased hydrogen levels in the blood}

Blood hydrogen levels of unoperated naïve animals ranged between $10.3 \pm 0.6 \mu \mathrm{mol} / \mathrm{L}$. Oral administration of $\mathrm{HW}$ increased blood hydrogen levels to $59.8 \pm 11.5 \mu \mathrm{mol} / \mathrm{L}$ in the arterial circulation and $48.7 \pm 4.2 \mu \mathrm{mol} / \mathrm{L}$ in the venous circulation $15 \mathrm{~min}$ after ingestion. Hydrogen levels were maintained at significantly increased levels for up to 30 min after ingestion when compared with animals given $\mathrm{RW}$ or DW, and returned to basal levels by $60 \mathrm{~min}$ after ingestion.
Oral administration of RW or DW did not change the concentration of hydrogen in the blood (Supplementary material online, Figure S1).

\subsection{Drinking HW reduced neointimal formation and macrophage infiltration in vein grafts}

Six weeks after the bypass procedure, a marked increase in $\mathrm{IH}$, consisting predominantly of $\alpha \mathrm{SMA}$-positive cells and collagen deposits, was seen in the rats that consumed RW or DW. In contrast, the rats that consumed HW exhibited significantly less $\mathrm{IH}$ (Figure $1 \mathrm{~A}$ and $B$ ). Additionally, there was marked macrophage infiltration, as indicated by the staining for ED-1-positive cells, 6 weeks after engraftment in the vein grafts treated with RW or DW, and macrophage infiltration was significantly reduced by HW consumption (Figure $1 A$ and $C$ ). 


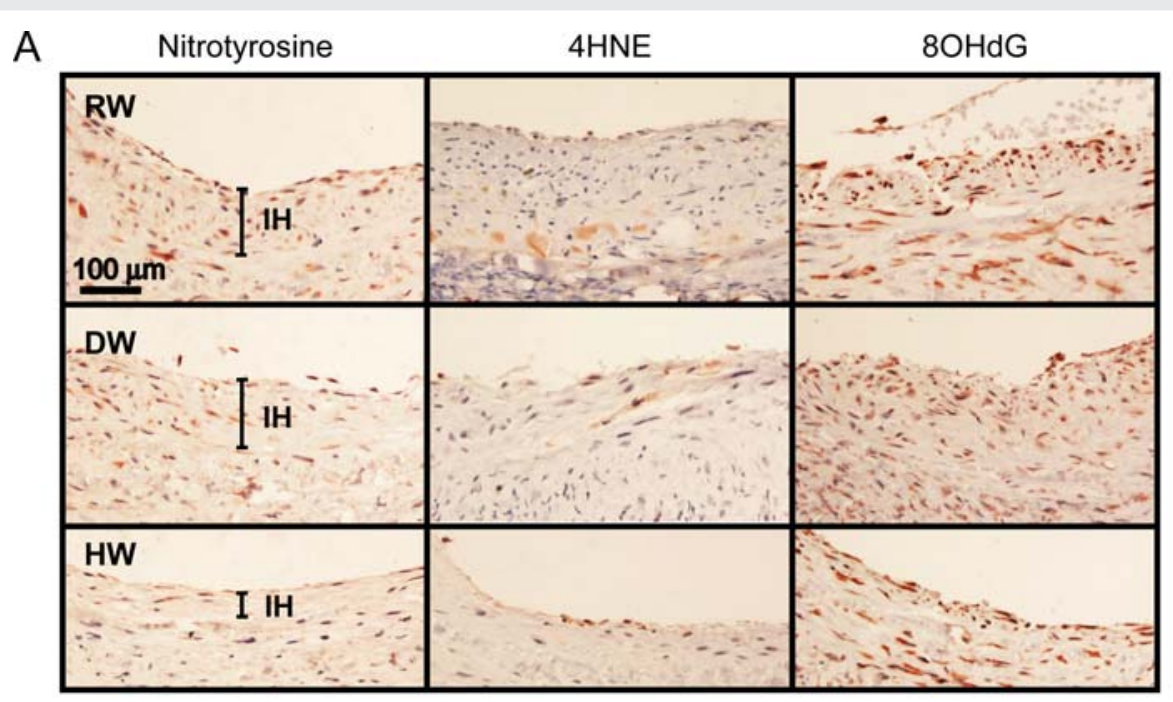

B

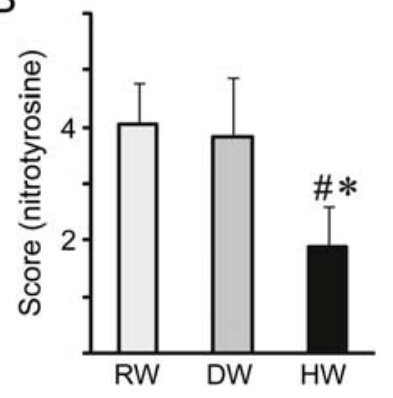

C

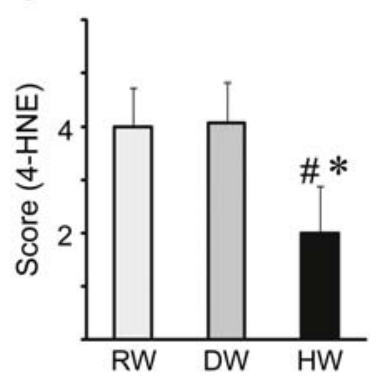

D

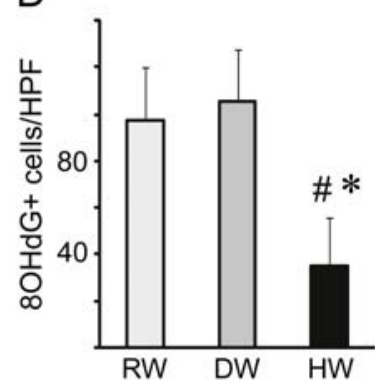

$\mathrm{E}$

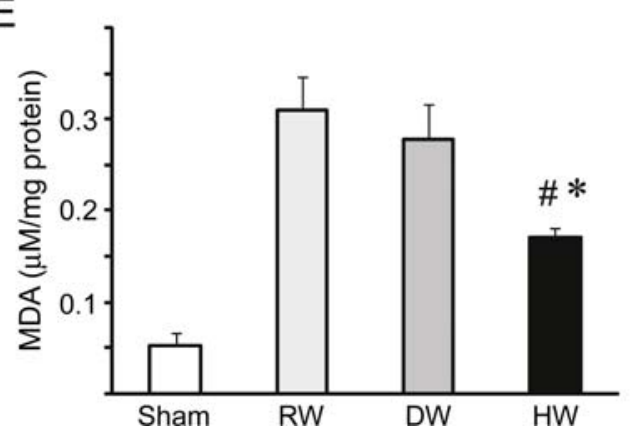

Figure 2 Evaluation of oxidative injury in the vein grafts. (A) Arterialized vein grafts taken 6 weeks after implantation were assessed using immunohistochemical stain for nitrotyrosine, 4HNE and 8-OHdG. Recipients were treated with RW, DW, or HW. Quantitation of staining intensity for (B) nitrotyrosine and $(C) 4-\mathrm{HNE}$. (D) The number of cells positive for $8-\mathrm{OHdG}(n=6$ per group; $5 \mathrm{HPF}$ were counted per sample). (E) MDA levels in the vein grafts ( $n=6$ per group, ${ }^{*} P<0.05$ vs. RW, ${ }^{\#} P<0.05$ vs. DW).

\subsection{Drinking HW reduced oxidative damage in vein grafts}

Because hydrogen reduces oxidative tissue damage in several experimental systems, markers for oxidative injury were assessed including nitrotyrosine, a stable end-product of tyrosine nitration by reactive nitrogen species, and $4 \mathrm{HNE}$, which is generated in the oxidation of lipids. Nitrotyrosine staining and 4-HNE adducts were prominent in the vein grafts in rats that consumed RW or DW. In contrast, staining for nitrotyrosine and 4-HNE was significantly attenuated in the vein grafts in rats that consumed HW (Figure $2 A-C$ ). Likewise, the number of cells containing $8-\mathrm{OHdG}$, a common product of nucleic acid oxidation, increased 6 weeks after engraftment in rats that consumed RW or DW. Drinking HW significantly decreased the number of $8-\mathrm{OHdG}$-positive cells (Figure 2A and D). The antioxidant activities of HW were confirmed by measurement of MDA, a marker of lipid peroxidation, in the vein grafts. Although MDA levels were elevated in the control vein grafts (RW or DW), oral intake of HW significantly reduced MDA levels after engraftment (Figure $2 E$ ).

\subsection{Hydrogen reduced endothelial denudation and local cell aggregation}

To further evaluate the changes in vein graft endothelial morphology, the grafts were harvested 1 week after implantation and 


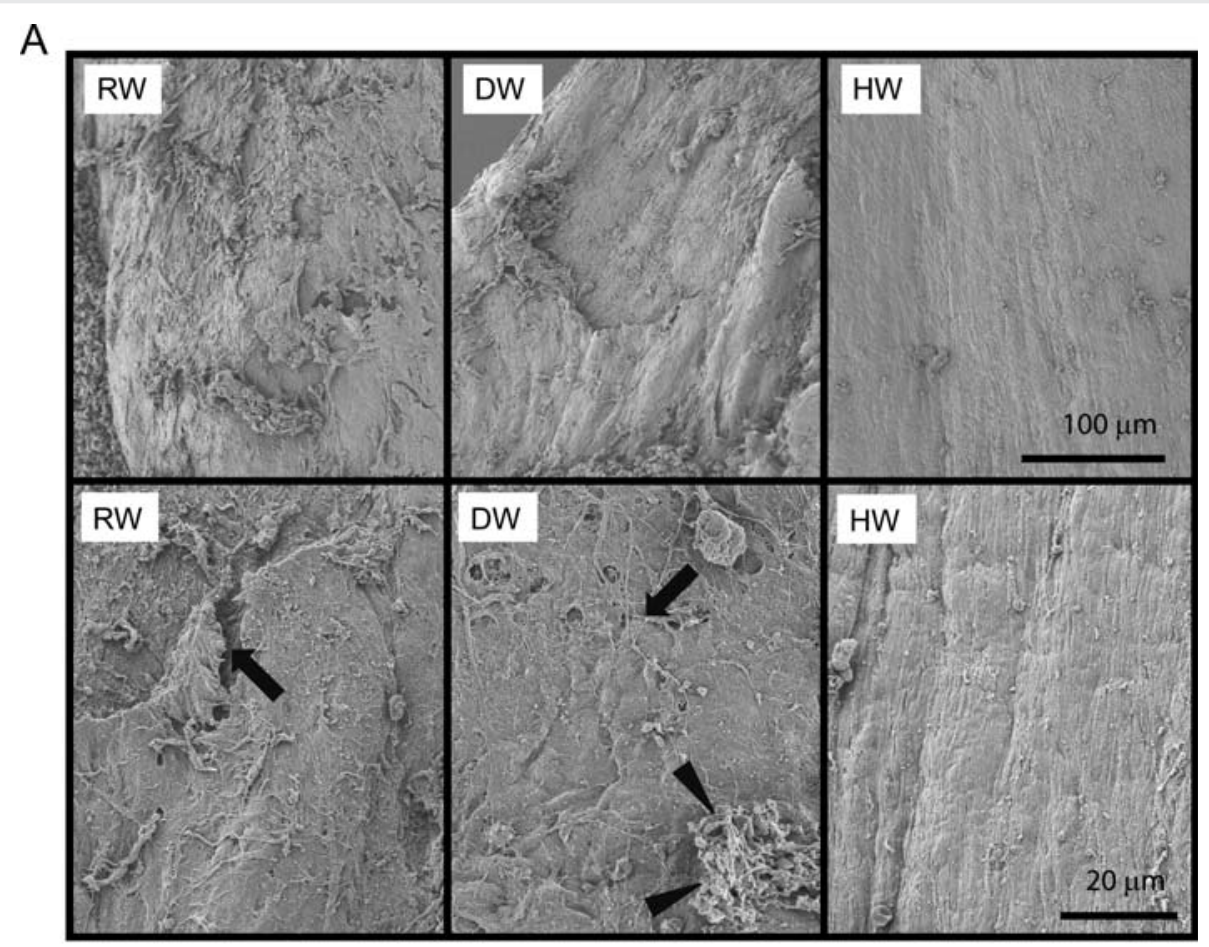

$\mathrm{B}$

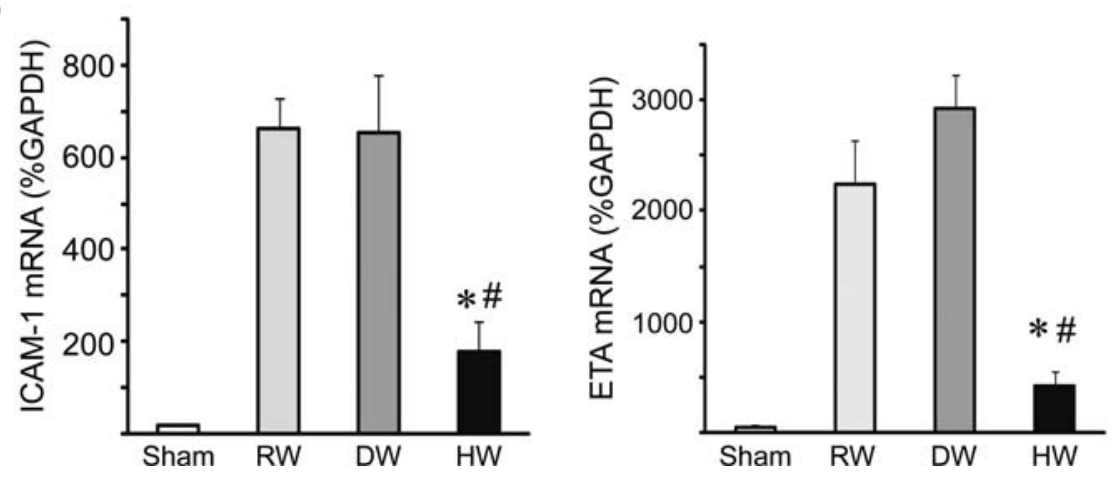

Figure 3 (A) SEM of the luminal surface of vein grafts taken 7 days after engraftment. Arrows indicate microthrombi; arrowheads indicate aggregated platelets. Images are representative of three vein grafts from each treatment group. (upper panels: lower magnification, lower panels: higher magnification) (B) Real-time RT-PCR for ICAM-1 and endothelin receptor-A (ETA). ( $n=5$ for each group, $* P<0.05$ vs. RW, ${ }^{\#} P<0.05$ vs. DW).

examined using SEM. In control vein grafts treated with RW or DW, SEM revealed significant endothelial denudation with the deposition of platelets, microthrombi, and leucocytes. These changes were completely attenuated in the vein grafts treated with HW; the vascular endothelial cells were well-preserved and had distinct junctions with few adherent platelets or leucocytes on the luminal surface (Figure $3 \mathrm{~A}$ ). Although the changes in endothelial denudation with the microthrombi seen in the RW and DW groups occurred only in certain patches, the images which we showed are representative and typically seen in all samples in each group. Because ICAM-1, endothelin-1, and endothelin receptors mediate vascular inflammation and $\mathrm{IH}$ formation, ICAM-1 and endothelin receptor-A mRNA expression was examined and was significantly up-regulated 7 days after engraftment in the vein grafts of rats treated with RW or DW. Less up-regulation of ICAM-1 and endothelin receptor-A occurred in the vein grafts of rats that received $\mathrm{HW}$ (Figure $3 \mathrm{~B}$ ).

\subsection{Hydrogen treatment reduced activation of p38 MAPK inflammatory cascades}

Phosphorylation of cytoplasmic p38MAPK, an important inflammatory mediator, was increased 1 week after surgery in the vein grafts of rats that received RW or DW. HW treatment suppressed $\mathrm{p} 38$ phosphorylation almost to the basal level. Hydrogen intake did not influence the phosphorylation of ERK1/2 (Figure 4A). In accordance with p38MAPK activation, mRNA levels for TNF- $\alpha$ and IL- 6 were increased in the grafts treated with RW or DW, and were significantly lower in the vein grafts treated with HW (Figure 4B).

\subsection{Hydrogen inhibited up-regulation of MMP-2 and MMP-9}

Because MMPs are important mediators of $\mathrm{H}$, and MMP-2 and MMP-9 promote neointima formation, we evaluated MMP expression 

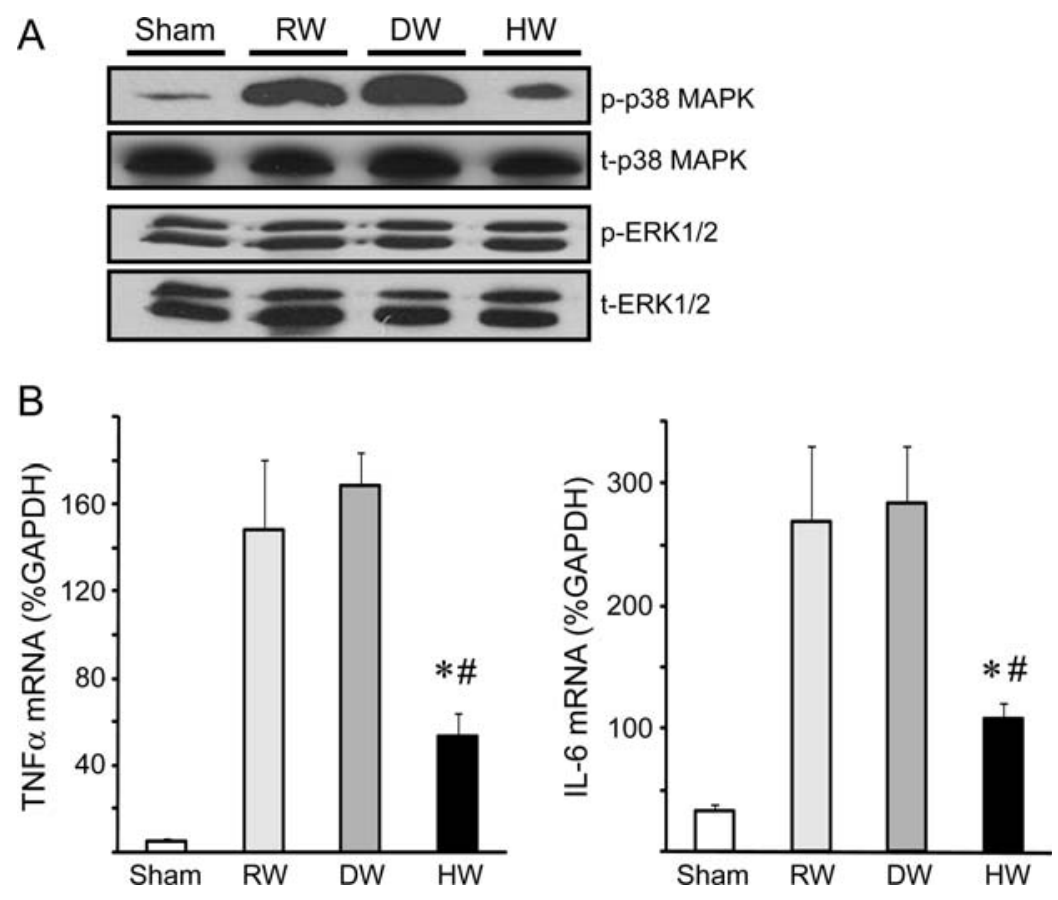

Figure 4 (A) Western blots for p38MAPK and ERK1/2 using the vein graft samples harvested 1 week after engraftment. (B) Real-time RT-PCR for TNF- $\alpha$ and IL-6. ( $n=5$ for each group, ${ }^{*} P<0.05$ vs. RW, ${ }^{\#} P<0.05$ vs. DW).

A

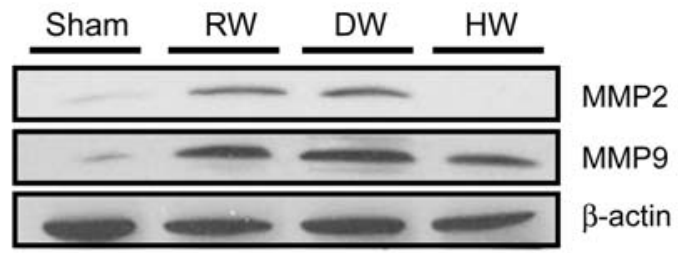

B
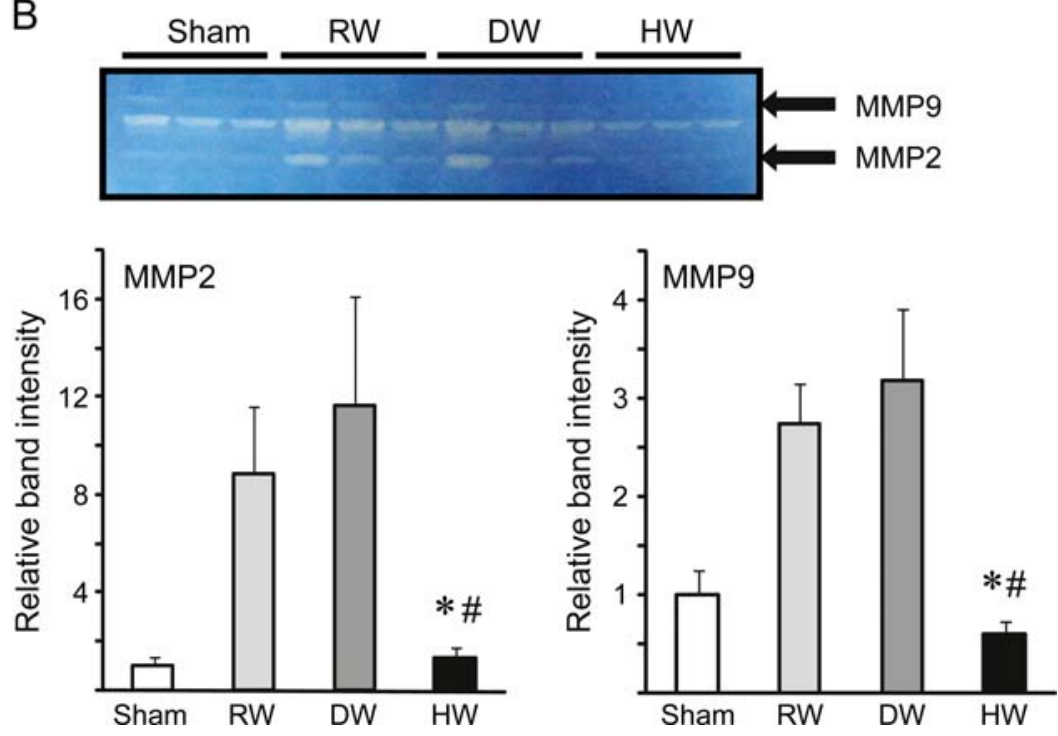

Figure 5 (A) Western blots for MMP-2 and MMP-9 using the vein graft samples harvested 1 week after engraftment. Images are representative of three independent experiments. (B) Enzymatic activity of MMP-2 and MMP-9 determined by zymography. $(n=3$ for each group, *P< 0.05 vs. RW, ${ }^{\#} P<0.05$ vs. DW). Histogram of band intensity of the bands for MMP-2 and MMP-9 zymogram of four different samples as measured by ImageJ computer software. 

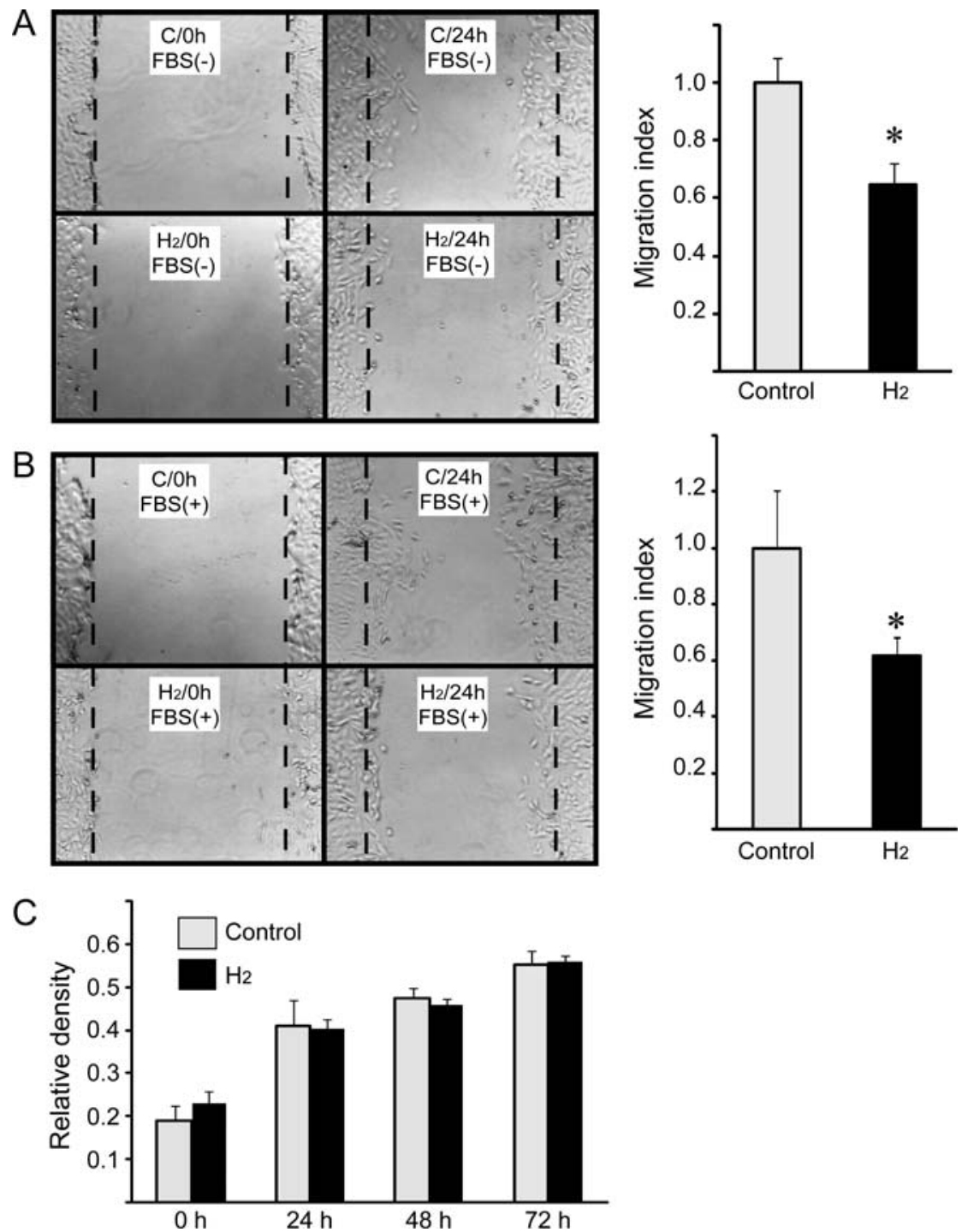

Figure 6 The wound migration assay $(A)$ without FBS and $(B)$ with FBS in the culture medium. Wounds in monolayers of A7r5 SMC cells. Representative images of three independent experiments are shown. Histograms depict average migration index. The original wound edge is marked with broken line (*P $<0.05$ vs. control). (C) Cell proliferation assay of cultured A7r5 SMC.

1 week after engraftment. MMP-2 and MMP-9 expression increased in the grafts of rats that received $\mathrm{RW}$ or $\mathrm{DW} .^{26-28} \mathrm{HW}$ consumption reduced the expression of both MMP-2 and MMP-9 (Figure 5A). A corresponding decrease in the enzymatic activities of MMP-2 and MMP-9 was confirmed using gelatin zymography. Both MMP-2 and MMP-9 activities were increased in the vein grafts in the recipients given RW or DW, and drinking HW significantly reduced these enzymatic activities (Figure 5B).

\subsection{SMC migration inhibition by hydrogen in vitro}

SMC migration and proliferation play important roles in the progression of $\mathrm{IH}$. To determine whether hydrogen had a direct effect on SMCs, the effects of hydrogen on SMC migration and proliferation were assessed in culture. In rat SMC cultures without hydrogen, cell migration was detected $24 \mathrm{~h}$ after wounding. Incubation in hydrogen-rich medium suppressed SMC migration even when foetal bovine serum was present (Figure $6 A$ and $B$ ), but did not affect SMC proliferation during $72 \mathrm{~h}$ of culture (Figure $6 \mathrm{C}$ ).

\subsection{Hydrogen protected endothelial cells from oxidative cellular injury}

The antioxidant effects of hydrogen on vascular endothelial cells were also examined using HUVECs. Exposure of HUVECs to $\mathrm{H}_{2} \mathrm{O}_{2}$ significantly reduced cellular viability and membrane integrity, as measured by increased LDH leakage. MDA levels in the culture significantly increased after $\mathrm{H}_{2} \mathrm{O}_{2}$ exposure, indicating cellular lipid peroxidation in response to $\mathrm{H}_{2} \mathrm{O}_{2}$ exposure. Incubation in hydrogen-rich medium significantly improved cell viability, reduced LDH leakage, and attenuated MDA production, indicating that hydrogen protected the HUVECs from cellular damage induced by $\mathrm{H}_{2} \mathrm{O}_{2}$ (Supplementary material online, Figure S1A-C). 


\section{Discussion}

In this study, we demonstrated that drinking HW significantly attenuated $\mathrm{IH}$ in a rat vein grafting model. This protection against $\mathrm{IH}$ included preservation of the structure of the endothelial layer and inhibition of oxidative stress, inflammation, and cell migration. This study clearly demonstrated that oral intake of hydrogen water can limit oxidative injury in vein grafts. Hydrogen also protected cultured HUVECs from oxidative damage after exposure to $\mathrm{H}_{2} \mathrm{O}_{2}$. Our results are consistent with a previous report that oral ingestion of hydrogensupplemented water ad libitum for 6 months prevented the development of atherosclerosis in apolipoprotein $E$ knockout mice that develop atherosclerosis quickly due to impaired cleaning of plasma lipoprotein, through hydrogen's ability to limit the deleterious effects of oxidative stress. ${ }^{29}$ Ohsawa et al. have advocated a role for hydrogen as a scavenger of ROS. ${ }^{13}$ Because the concentration of hydrogen is relatively low after oral intake of $\mathrm{HW}$, the scavenging properties of hydrogen may not play a major role in amelioration of $\mathrm{IH}$ in our study, although we do not exclude the possibility. ${ }^{17}$

Our data showed the fact that hydrogen has a very short half-life in the body of animals after oral intake. Currently, we lack a good explanation on how this brief transient elevation produces an effect. However, the blood concentration of hydrogen increased approximately five-fold over the basal levels, which is consistent with the previous study. ${ }^{17}$ We believe that this elevation from the basal level may activate signalling pathways that contribute to organ protection. These observations, along with other published data, suggest that hydrogen serves not only as an antioxidant, but also as a signalling molecule. ${ }^{30-33}$ The concentrations of hydrogen in tissues and blood, as well as intrinsic factors that affect hydrogen levels in vivo, are not fully understood, and it will be imperative to address these points to distinguish between the physiological and pharmacological effects of hydrogen.

$\mathrm{IH}$ is a consequence of vascular inflammation leading to SMC proliferation and migration, and synthesis of ECM. During $\mathrm{IH}$ formation, phosphorylation of p38 MAPK mediates inflammatory responses through regulation of the gene expression of a number of inflammatory cytokines and adhesion molecules, such as IL-6, TNF- $\alpha$, and ICAM-1. ${ }^{34-36}$ In our study, these inflammatory events were attenuated by hydrogen treatment, which is consistent with previous reports that drinking hydrogen-supplemented water suppresses inflammatory events during chronic allograft nephropathy through inhibition of MAPK activation. ${ }^{17}$ Likewise, Anggrahini et al. reported that endothelin-1 and its receptors mediate vascular inflammation and $\mathrm{IH}^{37}$ In our model, hydrogen treatment significantly reduced the expression of endothelin-1 receptor-A mRNA. In vein grafts, oxidative stress and inflammatory responses destroy the integrity of endothelial cell layer, thus inducing SMC proliferation and migration. In our SEM study, the vascular endothelial cell layer in hydrogentreated grafts was well-preserved with distinct junctions with few adherent platelets and leucocytes on the luminal surface.

Hydrogen reduced MMP-2 and MMP-9 expression and suppressed SMC migration. Cell migration is an integral component of $\mathrm{IH}$ development and requires ECM degradation and reorganization. MMPs play an important role in this process. ${ }^{26-28,38}$ In particular, MMP-2 and MMP-9 can enhance neointima formation, primarily by freeing SMCs from the cell-matrix contacts that normally restrict migration. ${ }^{27}$ Our data suggest that HW treatment inhibits SMC migration into the vein grafts at least partially by lowering the expression of MMP-2 and MMP-9 after engraftment. Inhibition of p38 MAPK decreases MMP2/9 expression in lung cancer cells ${ }^{39,40}$ and, therefore, decreased activation of P38 MAPK may lead to decreased MMP-2 and MMP-9 expression in the vein grafts after hydrogen treatment.

In summary, drinking $\mathrm{HW}$ can prevent the development of $\mathrm{IH}$, by reducing oxidative stress, inflammatory responses, and SMC migration. To our knowledge, this is the first study to show that hydrogen mitigates $\mathrm{IH}$ in arterialized vein grafts. Although the exact mechanisms need to be elucidated, hydrogen may have a huge potential as a safe and potent therapeutic medical gas. Further studies of hydrogen's mechanisms of action and studies towards clinical application in humans are certainly warranted.

\section{Supplementary material}

Supplementary material is available at Cardiovascular Research online.

\section{Acknowledgements}

The authors thank Lisa R. Chedwick and Jonathan M. Franks for technical support and Dr Shannon L. Wyszomierski for editing the manuscript.

\section{Conflict of interest: none declared.}

\section{Funding}

This work was supported by the China Scholarship Council (Q.S.), Daimaru Research Foundation (Q.S.), National Institutes of Health (R21 HL102528-01 to A.N.) and research funds of the Department of Cardiothoracic Surgery. T.K. is a recipient of the Thomas E. Starzl Postdoctoral fellowship.

\section{References}

1. Nguyen LL, Conte MS, Menard MT, Gravereaux EC, Chew DK, Donaldson MC et al. Infrainguinal vein bypass graft revision: factors affecting long-term outcome. J Vasc Surg 2004; 40:916-923.

2. Feinglass J, Kaushik S, Handel D, Kosifas A, Martin GJ, Pearce WH. Peripheral bypass surgery and amputation: Northern illinois demographics, 1993 to 1997. Arch Surg 2000; 135:75-80.

3. Lytle BW, Loop FD, Taylor PC, Simpfendorfer C, Kramer JR, Ratliff NB et al. Vein graft disease: the clinical impact of stenoses in saphenous vein bypass grafts to coronary arteries. J Thorac Cardiovasc Surg 1992;103:831-840.

4. Davies MG, Hagen PO. Pathophysiology of vein graft failure: a review. Eur J Vasc Endovasc Surg 1995;9:7-18.

5. Wallitt EJ, Jevon M, Hornick PI. Therapeutics of vein graft intimal hyperplasia: 100 years on. Ann Thorac Surg 2007;84:317-323.

6. Nakao A, Murase N, Ho C, Toyokawa H, Billiar TR, Kanno S. Biliverdin administration prevents the formation of intimal hyperplasia induced by vascular injury. Circulation 2005;112:587-591.

7. Nigri GR, Kossodo S, Waterman P, Fungaloi P, LaMuraglia GM. Free radical attenuation prevents thrombosis and enables photochemical inhibition of vein graft intimal hyperplasia. J Vasc Surg 2004;39:843-849.

8. Rosenbaum MA, Miyazaki K, Colles SM, Graham LM. Antioxidant therapy reverses impaired graft healing in hypercholesterolemic rabbits. J Vasc Surg 2010;51:184-193.

9. Liu SQ, Ruan YY, Tang D, Li YC, Goldman J, Zhong L. A possible role of initial cell death due to mechanical stretch in the regulation of subsequent cell proliferation in experimental vein grafts. Biomech Model Mechanobiol 2002;1:17-27.

10. Davies MG, Dalen H, Barber L, Svendsen E, Hagen PO. Lazaroid therapy (methylaminochroman: U83836e) reduces vein graft intimal hyperplasia. J Surg Res 1996;63 $128-136$.

11. Joddar B, Reen RK, Firstenberg MS, Varadharaj S, McCord JM, Zweier JL et al. Protandim attenuates intimal hyperplasia in human saphenous veins cultured ex vivo via a catalase-dependent pathway. Free Radic Biol Med 2011;50:700-709.

12. Huang CS, Kawamura T, Toyoda Y, Nakao A. Recent advances in hydrogen research as a therapeutic medical gas. Free Radic Res 2010;44:971-982.

13. Ohsawa I, Ishikawa M, Takahashi K, Watanabe M, Nishimaki K, Yamagata K et al. Hydrogen acts as a therapeutic antioxidant by selectively reducing cytotoxic oxygen radicals. Nat Med 2007;13:688-694.

14. Kajiya M, Silva MJ, Sato K, Ouhara K, Kawai T. Hydrogen mediates suppression of colon inflammation induced by dextran sodium sulfate. Biochem Biophys Res Commun 2009;386:11-15. 
15. Fujita K, Seike T, Yutsudo N, Ohno M, Yamada H, Yamaguchi $H$ et al. Hydrogen in drinking water reduces dopaminergic neuronal loss in the 1-methyl-4-phenyl-1,2,3,6-tetrahydropyridine mouse model of parkinson's disease. PLoS One 2009;4:e7247.

16. Abraini JH, Gardette-Chauffour MC, Martinez E, Rostain JC, Lemaire C. Psychophysiological reactions in humans during an open sea dive to $500 \mathrm{~m}$ with a hydrogen-helium-oxygen mixture. J Appl Physiol 1994;76:1113-1118.

17. Cardinal JS, Zhan J, Wang Y, Sugimoto R, Tsung A, McCurry KR et al. Oral hydrogen water prevents chronic allograft nephropathy in rats. Kidney Int 2010;77:101-109.

18. Kamimura N, Nishimaki K, Ohsawa I, Ohta S. Molecular hydrogen improves obesity and diabetes by inducing hepatic fgf 21 and stimulating energy metabolism in $\mathrm{db} / \mathrm{db}$ mice. Obesity 2011;19:1396-1403.

19. Nakao A, Toyoda Y, Sharma P, Evans M, Guthrie N. Effectiveness of hydrogen rich water on antioxidant status of subjects with potential metabolic syndrome-an open label pilot study. J Clin Biochem Nutr 2010;46:140-149.

20. Kajiyama S, Hasegawa G, Asano M, Hosoda H, Fukui M, Nakamura N et al. Supplementation of hydrogen-rich water improves lipid and glucose metabolism in patients with type 2 diabetes or impaired glucose tolerance. Nutr Res 2008;28:137-143.

21. Nakao A, Huang CS, Stolz DB, Wang Y, Franks JM, Tochigi N et al. Ex vivo carbon monoxide delivery inhibits intimal hyperplasia in arterialized vein grafts. Cardiovasc Res 2010;89:457-463.

22. Buchholz BM, Kaczorowski DJ, Sugimoto R, Yang R, Wang Y, Billiar TR et al. Hydrogen inhalation ameliorates oxidative stress in transplantation induced intestinal graft injury. Am J Transplant 2008;8:2015-2024.

23. Sun H, Valdivia LA, Subbotin V, Aitouche A, Fung J], Starzl TE et al. Improved surgical technique for the establishment of a murine model of aortic transplantation. Microsurgery 1998;18:368-371.

24. Cheng ST, Chen ZF, Chen GQ. The expression of cross-linked elastin by rabbit blood vessel smooth muscle cells cultured in polyhydroxyalkanoate scaffolds. Biomaterials 2008;29:4187-4194.

25. Wei Y, Chen L, Chen J, Ge L, He RQ. Rapid glycation with d-ribose induces globular amyloid-like aggregations of BSA with high cytotoxicity to sh-sy5y cells. BMC Cell Biol 2009;10:10.

26. Berceli SA, Jiang Z, Klingman NV, Pfahnl CL, Abouhamze ZS, Frase CD et al. Differential expression and activity of matrix metalloproteinases during flow-modulated vein graft remodeling. J Vasc Surg 2004;39:1084-1090.

27. Thomas AC, Newby AC. Effect of matrix metalloproteinase- 9 knockout on vein graft remodelling in mice. J Vasc Res 2010;47:299-308.
28. Zhang WD, Bai HZ, Sawa Y, Yamakawa T, Kadoba K, Taniguchi K et al. Association of smooth muscle cell phenotypic modulation with extracellular matrix alterations during neointima formation in rabbit vein grafts. J Vasc Surg 1999;30:169-183.

29. Ohsawa I, Nishimaki K, Yamagata K, Ishikawa M, Ohta S. Consumption of hydrogen water prevents atherosclerosis in apolipoprotein e knockout mice. Biochem Biophys Res Commun 2008;377:1195-1198.

30. Itoh T, Fujita Y, Ito M, Masuda A, Ohno K, Ichihara M et al. Molecular hydrogen suppresses fcepsilonri-mediated signal transduction and prevents degranulation of mast cells. Biochem Biophys Res Commun 2009;389:651-656.

31. Kawamura T, Huang CS, Tochigi N, Lee S, Shigemura N, Billiar TR et al. Inhaled hydrogen gas therapy for prevention of lung transplant-induced ischemia/reperfusion injury in rats. Transplantation 2010;90:1344-1351.

32. Huang CS, Kawamura T, Peng X, Tochigi N, Shigemura N, Billiar TR et al. Hydrogen inhalation reduced epithelial apoptosis in ventilator-induced lung injury via a mechanism involving nuclear factor-kappa b activation. Biochem Biophys Res Commun 2011; 408:253-258.

33. Buchholz BM, Masutani K, Kawamura T, Peng X, Toyoda Y, Billiar TR et al. Hydrogen-enriched preservation protects the isogeneic intestinal graft and amends recipient gastric function during transplantation. Transplantation 2011;92:985-992.

34. Ono K, Han J. The p38 signal transduction pathway: activation and function. Cell Signal 2000;12:1-13.

35. Jiang Z, Shukla A, Miller BL, Espino DR, Tao M, Berceli SA et al. Tumor necrosis factor-alpha and the early vein graft. J Vasc Surg 2007;45:169-176.

36. Chello M, Mastroroberto P, Frati G, Patti G, D’Ambrosio A, Di Sciascio G et al. Pressure distension stimulates the expression of endothelial adhesion molecules in the human saphenous vein graft. Ann Thorac Surg 2003;76:453-458.

37. Anggrahini DW, Emoto N, Nakayama K, Widyantoro B, Adiarto S, Iwasa $\mathrm{N}$ et al. Vascular endothelial cell-derived endothelin-1 mediates vascular inflammation and neointima formation following blood flow cessation. Cardiovasc Res 2009;82:143-151.

38. Sharony R, Pintucci G, Saunders PC, Grossi EA, Baumann FG, Galloway AC et al. Matrix metalloproteinase expression in vein grafts: role of inflammatory mediators and extracellular signal-regulated kinases-1 and -2. Am J Physiol Heart Circ Physiol 2006;290:H1651-H1659.

39. Woo MS, Jung SH, Kim SY, Hyun JW, Ko KH, Kim WK et al. Curcumin suppresses phorbol ester-induced matrix metalloproteinase- 9 expression by inhibiting the PKC to MAPK signaling pathways in human astroglioma cells. Biochem Biophys Res Commun 2005;335:1017-1025

40. Chien ST, Lin SS, Wang CK, Lee YB, Chen KS, Fong $Y$ et al. Acacetin inhibits the invasion and migration of human non-small cell lung cancer a549 cells by suppressing the p38alpha mapk signaling pathway. Mol Cell Biochem 2011;350:135-148. 